University of Minnesota Morris Digital Well

University of Minnesota Morris Digital Well

$10-2005$

\title{
Pure Nash Equilibria of Coordination Matrix Games
}

David P. Roberts

University of Minnesota - Morris, roberts@morris.umn.edu

Follow this and additional works at: https://digitalcommons.morris.umn.edu/mathematics

Part of the Mathematics Commons

\section{Recommended Citation}

David P. Roberts. Pure Nash equilibria of coordination matrix games. Economics Letters 89 (2005), no. 1, 7-11.

This Article is brought to you for free and open access by the Faculty and Staff Scholarship at University of Minnesota Morris Digital Well. It has been accepted for inclusion in Mathematics Publications by an authorized administrator of University of Minnesota Morris Digital Well. For more information, please contact skulann@morris.umn.edu. 


\title{
PURE NASH EQUILIBRIA OF COORDINATION MATRIX GAMES
}

\author{
DAVID P. ROBERTS \\ DIVISION OF SCIENCE AND MATHEMATICS, UNIVERSITY OF MINNESOTA-MORRIS
}

\author{
Abstract. We give a simple formula for the chance that a random $m$-by- $n$ coor- \\ dination game has exactly $k$ pure Nash equilibria and compare the payoffs at the \\ different equilibria. \\ Keywords: pure Nash equilibrium, coordination game \\ JEL classification: $\mathrm{C} 72$
}

\section{INTRODUCTION}

Stanford (1999) studied in this journal the number of pure Nash equilibria in random $m$-by- $n$ coordination games. In Section 2, we considerably sharpen Stanford's main results, getting a simple formula for the chance that a random $m$-by- $n$ coordination game has exactly $k$ pure Nash equilibria. In Section 3, we complement our result by discussing how the payoffs at the different equilibria are typically quite different from one another.

Our mathematical setting throughout this letter involves a Player 1 choosing among $m$ strategies and a Player 2 choosing among $n$ strategies. If Player 1 chooses his $i^{\text {th }}$ strategy and Player 2 chooses his $j^{\text {th }}$ strategy then both players receive a return of $a_{i j}$. Thus to give an $m$-by- $n$ coordination game between Player 1 and Player 2 is to give the $m$-by- $n$ matrix $A=\left(a_{i j}\right)$. To avoid complications, we assume throughout that the game is strict in the sense that all the $a_{i j}$ are distinct. This allows us to also use the alternative notation $a(1), a(2), \ldots, a(m n)$, where the $a(r)$ are the $a_{i j}$ listed in descending order.

A pure Nash equilibrium for $A$ is a pair $(i, j)$ such that $a_{i j}$ is both the largest entry in its row and the largest entry in its column. It will be convenient to refer to the number $a_{i j}$ itself as the pure Nash equilibrium. We can use such language without any ambiguity because of our strictness assumption.

Clearly the largest entry $a(1)$ is a pure Nash equilibrium. Equally clearly, optimal coordinated play by Player 1 and 2 would result in this equilibrium being chosen. However, of course, our simple situation is part of a number of more complicated models of economic behavior. The two players may not be in good communication. They may be distrustful of one another. They may be playing repeated games, perhaps making their choices via some evolutionary algorithm.

In all the situations just listed, the presence of other pure Nash equilibria can be viewed as obstructions to $a(1)$ being chosen. It is thus important to have a good understanding of the other pure Nash equilibria and their associated payoffs $a(r)$; these topics correspond to our Sections 2 and 3 respectively. For more on the economic importance of coordination games and their Nash equilibria, we refer the reader to the first section of Stanford's letter.

Tel.: 320-589-6348; Fax.: 320-589-6371

Address: Division of Science and Math; University of Minnesota-Morris; Morris, MN 56267; USA

E-mail address: roberts@morris.umn.edu 


\section{The Number of PURE EQUILIBRIA}

Clearly the pure Nash equilibria of a coordination game $A$ depend on the entries $a_{i j}$ only through how they are ordered. Following Stanford, we consider random games where the $(m n)$ ! possible orderings occur with equal frequency.

Let $A$ be an $m$-by- $n$ coordination game. Let $N$ be its set of pure Nash equilibria. For every subset $S$ of $N$ let $W(S)$ be the smallest minor of $A$ containing $S$. We call a $w$-by- $w$ minor full iff it arises in this way. Our terminology captures the fact that a $w$-by- $w$ minor of $A$ contains $\leq w$ of $A$ 's pure Nash equilibria, with equality iff $A$ is full.

Our main statement has two parts. The two parts together refine Stanford's main results, as we'll explain after the proof.

Theorem 1. Let $e_{m, n, k}$ be the chance that a random $m$-by-n coordination game has exactly $k$ pure Nash equilibria. Then

$$
e_{m, n, k}=\left(\begin{array}{c}
m \\
k
\end{array}\right)\left(\begin{array}{l}
n \\
k
\end{array}\right) \frac{k(m-1) !(n-1) !}{(m+n-1) !} .
$$

Let $E_{m, n, w}$ be the average number of full $w$-by-w minors in random $m$-by-n coordination games. Then

$$
E_{m, n, w}=\left(\begin{array}{l}
m \\
w
\end{array}\right)\left(\begin{array}{l}
n \\
w
\end{array}\right) \frac{(m+n-w-1) ! w !}{(m+n-1) !} .
$$

Proof. In general, suppose $e_{0}, e_{1}, \ldots, e_{d}$ is a given finite sequence of numbers. Introduce the polynomial $f(x)=\sum_{k=0}^{d} e_{k} x^{k}$. Define a second sequence $E_{0}, \ldots, E_{d}$ by $f(x)=$ $\sum_{w=0}^{d} E_{w}(x-1)^{w}$. Comparing coefficients, one has

$$
\begin{aligned}
E_{w} & =\sum_{k=w}^{d}\left(\begin{array}{l}
k \\
w
\end{array}\right) e_{k}, \\
e_{k} & =\sum_{w=k}^{d}\left(\begin{array}{l}
w \\
k
\end{array}\right)(-1)^{w-k} E_{w} .
\end{aligned}
$$

We say that the row vector $E=\left(E_{0}, E_{1}, \ldots, E_{d}\right)$ is the Pascal transform of the row vector $e=\left(e_{0}, e_{1}, \ldots, e_{d}\right)$ and that $e$ is the inverse Pascal transform of $E$. Our terminology reflects the fact that if one writes (3) in matrix form as $E=e P$, then $P$ is lower triangular with the lower triangle being exactly Row 0 through Row $d$ of Pascal's triangle.

For the proof of (1) and (2), we view $m$ and $n$ as fixed and both $k$ and $w$ as running from 0 to $d=\min (m, n)$. For $k=0$, both sides of (1) are 0 . For $w=0$, both sides of (2) are 1 ; for on the left side, any $m$-by- $n$ matrix $A$ has exactly one 0 -by-0 minor and this minor certainly contains 0 Nash equilibria.

Our proof of (1) has three steps. Step 1 is to observe that the vector $E_{m, n}=$ $\left(1, E_{m, n, 1}, \ldots, E_{m, n, d}\right)$ is the Pascal transform of the vector $e_{m, n}=\left(0, e_{m, n, 1}, \ldots, e_{m, n, d}\right)$. Step 2 is to prove (2) by a direct argument. Step 3 is to apply a classical fact to prove that the right side of (1) is the inverse Pascal transform of the right side of (2). The three steps are completely independent of one another and can therefore be read in any order.

Step 1. From the definition of full, it is clear that a game with $k$ pure Nash equilibria has $\left(\begin{array}{c}k \\ w\end{array}\right)$ full $w$-by- $w$ minors. Combined with the definition (3), this observation proves that $E_{m, n}$ is indeed the Pascal transform of $e_{m, n}$.

Step 2. Let $E_{m, n, w}^{\prime}$ be the chance that a random $m$-by- $n$ coordination game $A$ satisfies $\left(^{*}\right), a_{11}>a_{22}>\cdots>a_{w w}$, and $\left(^{* *}\right)$, all $w$ of these $a_{u u}$ are pure Nash equilibria. Then

$$
E_{m, n, w}=\left(\begin{array}{l}
m \\
w
\end{array}\right)\left(\begin{array}{l}
n \\
w
\end{array}\right) w !^{2} E_{m, n, w}^{\prime}
$$


For, in general, contributions to $E_{m, n, w}$ come from $a_{i_{1}, j_{1}}>\cdots>a_{i_{w}, j_{w}}$, with each $a_{i_{u}, j_{u}}$ a Nash equilibrium. The factor $\left(\begin{array}{c}m \\ w\end{array}\right) w$ ! in (5) comes from our focus on the special case $i_{u}=u$ and the factor $\left(\begin{array}{c}n \\ w\end{array}\right) w$ ! similarly comes from our focus on the special case $j_{u}=u$.

Now Conditions $(*)$ and $\left({ }^{* *}\right)$ on $A$ can be reformulated as $w$ independent conditions. Namely the $t^{\text {th }}$ condition is that $a_{w+1-t, w+1-t}$ is the largest of the $(m+n-t) t$ numbers $a_{i j}$ with indices satisfying $0 \leq w-i \leq t-1$ or $0 \leq w-j \leq t-1$. Thus one has

$$
E_{m, n, w}^{\prime}=\prod_{t=1}^{w} \frac{1}{(m+n-t) t}=\frac{(m+n-w-1) !}{w !(m+n-1) !} .
$$

Inserting (6) into (5) and canceling a $w$ ! gives (2).

Step 3. Let

$$
J_{d}^{a, b}(x)=(-1)^{d}\left(\begin{array}{c}
b-a \\
d
\end{array}\right)^{-1} P_{d}^{a, b}(2 x-1)=\sum_{k=0}^{d} e_{k}^{a, b} x^{k}=\sum_{w=0}^{d} E_{w}^{a, b}(x-1)^{w} .
$$

Here the $P_{d}^{a, b}(y)$ are the classically normalized Jacobi polynomials with parameters $a$ and $b$, while the $J_{d}^{a, b}(x)$ are renormalized versions more suited to our context. Then

$$
\begin{aligned}
e_{k}^{a, b} & =\left(\begin{array}{c}
b-a \\
d
\end{array}\right)^{-1}\left(\begin{array}{c}
d+b \\
d-k
\end{array}\right)\left(\begin{array}{c}
-1-a-b-d \\
k
\end{array}\right), \\
E_{w}^{a, b} & =\left(\begin{array}{c}
b-a \\
d
\end{array}\right)^{-1}\left(\begin{array}{c}
-a-w-1 \\
d-w
\end{array}\right)\left(\begin{array}{c}
-1-a-b-d \\
w
\end{array}\right),
\end{aligned}
$$

from e.g. Equations 8.960 and 8.961.1 of Gradshteyn and Ryzhik (1994) and the general formula $\left(\begin{array}{c}-a \\ b\end{array}\right)=(-1)^{b}\left(\begin{array}{c}a+b-1 \\ b\end{array}\right)$. Then, treating the cases $m \geq n$ and $n \geq m$ separately, one can check that $e_{k}^{-m-n,-1}$ and $E_{w}^{-m-n,-1}$ simplify to the right sides of (1) and (2) respectively, by direct cancelation of factorials. So the right side of (1) is indeed the inverse Pascal transform of the right side of (2).

Stanford proves in his Theorem 1.1 that the expected number of pure Nash equilibria is $m n /(m+n-1)$. He proves in his Theorems 1.2 and 1.3 results indicating that the distribution about this mean is tight. He writes on page 31 that a more "complete analysis" would be desirable, but is obstructed by "complications." Equations (2) and (3) with $w=1$ say that the expected number of pure Nash equilibria is $\langle k\rangle=E_{m, n, 1}=$ $m n /(m+n-1)$, recovering Stanford's Theorem 1.1. Equations (2) and (3) now for $w \leq 2$ give the central variance of the number of pure Nash equilibria as

$$
\left\langle k^{2}\right\rangle-\langle k\rangle^{2}=\left(2 E_{m, n, 2}+E_{m, n, 1}\right)-\left(E_{m, n, 1}^{2}\right)=\frac{m(m-1) n(n-1)}{(m+n-1)^{2}(m+n-2)} ;
$$

this is a much sharper statement than the inequality in Stanford's Theorem 1.2. The full statement of our Theorem 1 is the desired complete analysis. In particular, it is immediate from (1) that for fixed $k$, one has $e_{m, n, k} \rightarrow 0$ as $\min (m, n) \rightarrow \infty$, and this is enough to recover Stanford's Theorem 1.3.

Remarkably, the quantities appearing in Theorem 1 also appear in the study of random games where the $a_{i j}$ are chosen independently with respect to the Cauchy density $f(x)=1 /\left(\pi\left(x^{2}+1\right)\right)$. Namely, according to Theorem 1 of Roberts $(2004), e_{m, n, k}$ is also the chance that the unique mixed Nash equilibrium of a Cauchy-random $m$-by- $n$ zero-sum game is $k$-by- $k$. Similarly, according now to Theorem 2 of Roberts (2004), for $w \geq 1$ the quantity $E_{m, n, w}$ is also the expected number of $w$-by- $w$ mixed Nash equilibria of a Cauchy-random $m$-by- $n$ coordination game. The asymptotics of both $e_{m, n, k}$ and $E_{m, n, w}$ are studied at some length in Roberts (2004).

There is a second connection between the $E_{m, n, w}$ and mixed Nash equilibria. Namely suppose given a matrix $A$. Redefine each $a(r)$ to be $b^{m n-r}$, with $b>1$ an as yet 
unspecified number. Note that the order of the matrix entries is not changed in this process, but as $b$ gets large the preference for a given return $a(r)$ over the next $a(r+1)$ increases. We can prove that for $b$ sufficiently large, the minors coming from mixed Nash equilibria are exactly the full minors. So in this "strong preference limit," (2) gives the expected number of $w$-by- $w$ mixed Nash equilibria.

\section{PAyoffs AT PURE EQUilibria}

Let $A[1], \ldots, A[k]$ be the pure Nash equilibria of $A$ in descending order. As explained in Section 1, it is important to understand how much smaller than $A[1]$ the other $A[u]$ are. There are two separate effects which can contribute to $A[u]$ being much smaller than $A[1]$. First, the $a(1), a(2), \ldots$, can decrease rapidly. Second, in the equality $A[u]=a(r)$, one may have $r$ larger than $u$.

To pursue the first effect, we consider probability densities $f$ on the real line and consider the real numbers $a_{i j}$ drawn independently with respect to $f$. The strength of the first effect depends on right tail of $f$. Any number of statements can be established using the results in Chapter 10 of David and Nagaraja (2003). As an example including the Cauchy density, suppose $f(x) \sim C / x^{2}$ as $x \rightarrow \infty$, for some constant $C>0$. Then the chance that $\log (a(r) / a(r+1))$ is in $[c, d]$ tends to $r \int_{c}^{d} e^{-r x} d x$ as $m n \rightarrow \infty$. In particular, the asymptotic median value of $a(r) / a(r+1)$ is $2^{1 / r}$ while the asymptotic mean is infinite for $r=1$ and equal to $r /(r-1)$ for $r \geq 2$. Thus, in this heavy tail setting, $a(1)$ is very much preferred over $a(2)$, which is still substantially preferred over $a(3)$, and so on.

To treat the second effect, let $r_{m, n}(u)$ be the average $r$ in the equality $A[u]=a(r)$, as $A$ ranges over games with at least $u$ pure Nash equilibria. We have an exact formula for $r_{m, n}(u)$ but it is too complicated to give economic insight. Instead we give two limiting statements which can be established without using the whole formula:

$$
\begin{aligned}
\lim _{n \rightarrow \infty} r_{m, n}(u) & =u+\sum_{i=1}^{u-1} \frac{i}{m-i}, \\
\lim _{u \rightarrow \infty}\left(\frac{r_{u, u}(u)}{u}-\frac{\log (2 u)+\gamma}{2}\right) & =0 .
\end{aligned}
$$

In the second statement, $\gamma \approx 0.5772$ is Euler's constant. Each statement shows that $r_{m, n}(u)$ can be quite a bit larger than $u$.

\section{REFERENCES}

[1] David, H. A. and H. N. Nagaraja, 2003, Order statistics, third edition (Wiley).

[2] Gradshteyn, I. S. and I. M. Ryzhik, with A. Jeffrey, editor, 1994, Table of integrals, series, and products, fifth edition (Academic Press).

[3] Roberts, D. P., 2004, Nash equilibria of Cauchy-random zero-sum and coordination matrix games, submitted, 12 pages. Posted at http://cda.morris.umn.edu/ roberts

[4] Stanford, W., 1999, On the number of pure strategy Nash equilibria in finite common payoffs games, Economics Letters 62, 29-34 